\title{
Luminescent Qdots for Molecular Imaging and Traceable Drug Delivery
}

Xiaohu Gao

Department of Bioengineering, University of Washington, Seattle, WA 98195

Metal and semiconductor nanoparticles in the 1-10 $\mathrm{nm}$ size range are of considerable current interest, not only because of their unique size-dependent properties but also their dimensional similarities with biological macromolecules (e.g., nucleic acids and proteins). These similarities could allow an integration of nanotechnology and biology, leading to major advances in medical diagnostics, prognostics, and targeted therapeutics. In this talk, I present recent development of multifunctional nanostructures for biomedical applications, such as bioconjugated nanoparticles for tumor molecular imaging, profiling, and treatment. 